\begin{abstract}
¿Cómo citar el artículo?
Rojas Arenas, I. D., Vélez Castañeda, Ch. K., Durango Marín, J. A., Díaz Peláez, A. y Rodríguez Álvarez, A. F. (mayo-agosto, 2020). Percepción del proceso de formación por competencias y su relación con las prácticas empresariales: un caso de estudio. Revista Virtual Universidad Católica del Norte,

(60), 46-68., https://www.doi.org/10.35575/rvucn.n60a4
\end{abstract}

\title{
| Percepción del proceso de formación por competencias y su relación con las prácticas empresariales: un caso de estudio ${ }^{1}$
}

\author{
Perception of the competence-based training process and its relation to \\ business internship: a case study

\section{Iván Darío Rojas Arenas} \\ Doctorando en Pensamiento Complejo \\ Institución Universitaria Pascual Bravo \\ Medellín, Colombia \\ ivan.rojasar@pascualbravo.edu.co \\ Orcid: https://orcid.org/0000-0002-9300-3176 \\ Chárol Kátherin Vélez Castañeda \\ Magister en Logística Integral \\ Institución Universitaria Pascual Bravo \\ Medellin, Colombia \\ charol.velez@pascualbravo.edu.co \\ Orcid: https://orcid.org/0000-0003-2383-0560
}

\section{José Alejandro Durango Marín}

Magister en Administración Institución Universitaria Pascual Bravo Medellín, Colombia ja.durango@pascualbravo.edu.co Orcid: https://orcid.org/0000-0002-7231-8689

\section{Andrés Felipe Rodríguez Álvarez}

Magister en Ingeniería de Sistemas

Corporación Universitaria UniSabaneta

Medellín, Colombia

andres.rodriguez.docente@unisabaneta.edu.co

orcid: https://orcid.org/0000-0001-6301-3093

\author{
Alejandro Díaz Peláez \\ Doctorando en Administración \\ Institución Universitaria Pascual Bravo \\ Medellín, Colombia \\ a.diazpe@pascualbravo.edu.co \\ Orcid: https://orcid.org/0000-0002-6009-6812
}

Recibido: 28 de octubre de 2019 Evaluado: 17 de febrero de 2020

Aprobado: 28 de abril de 2020

Tipo de artículo: Investigación científica y tecnológica.

\section{| Resumen}

Las prácticas profesionales son una forma de validar la pertinencia de los programas académicos, al evaluar el desempeño de los practicantes en entornos reales en donde se requiere la aplicación del conocimiento adquirido; en este sentido, el presente artículo tiene como objetivo hacer un análisis acerca de la percepción de las competencias desarrolladas por practicantes de Tecnología en Producción Industrial e Ingeniería Industrial, de una institución universitaria de la ciudad de Medellín. Para tal efecto, se hizo un comparativo de la percepción de estudiantes, docentes y empresas, en relación con las competencias del Ser, el Saber y el Saber-Hacer, lo cual se complementó con la encuesta institucional desarrollada por la oficina de prácticas de dicha institución, a partir de una metodología descriptiva con enfoque mixto, no experimental, transeccional - correlacional. Como resultado del estudio estadístico se

1 Articulo derivado del proyecto "Análisis de las prácticas empresariales en los programas de Tecnología en Producción Industrial e Ingeniería Industrial de la I. U. Pascual Bravo", financiado por la Institución Universitaria Pascual Bravo. 
rechazó la hipótesis planteada para los grupos de interés, lo cual implica que existen diferencias en la percepción de estos, frente al desarrollo de competencias profesionales y su aplicación en las organizaciones. Se resalta como conclusión, para la población estudiada, la importancia de la relación entre las competencias adquiridas en el proceso formativo y un adecuado desempeño en la práctica profesional.

Palabras clave: Competencias laborales; Desempeño profesional; Evaluación por competencias; Formación por competencias; Pertinencia social; Relación universidad-empresa-estado.

\section{| Abstract}

Professional practices are a way to validate the relevance of academic programs by evaluating the performance of practitioners in real environments where the application of acquired knowledge is required; in this sense, this article aims to make an analysis of the perception of the competencies developed by practitioners of Technology in Industrial Production and Industrial Engineering at a university institution in the city of Medellin. For this purpose, a comparison was made of the perception of students, teachers and companies in relation to the competencies of Being, Knowledge and Know-how, which was complemented with the institutional survey developed by the practice office of that institution, based on a descriptive methodology with a mixed approach, non-experimental, transectional - correlational. As a result of the statistical study, the hypothesis raised for the stakeholders was rejected, which implies that there are differences in the perception of the stakeholders regarding the development of professional competencies and their application in the organizations. The importance of the relationship between the competencies acquired in the training process and adequate performance in professional practice is highlighted as a conclusion for the population studied.

Palabras clave: Labour skills; Professional performance; Assessment by skills; Training by skills; Social relevance; University-industry-state relationship.

\section{| Introducción}

El acelerado ritmo de cambio que viven las empresas en la actualidad, inmersas en un ambiente competitivo mediado por el vertiginoso avance tecnológico, ha generado un fuerte impacto en las organizaciones, la sociedad y los modelos educativos; asimismo, la difusión del conocimiento es cada vez más veloz, haciendo que las instituciones de educación superior -IES- deban validar constantemente sus procesos de enseñanza - aprendizaje, actualizando sus planes de estudio y metodologías empleadas (Vera, Álava y Basurto, 2019). Además, la formación por competencias requiere hoy más que nunca una adecuada interrelación entre IES y empresas, quienes validan la pertinencia del conocimiento adquirido en el proceso educativo, a través de las prácticas profesionales (Ronquillo, Cabrera y Barberán, 2019). Así las cosas, es claro que una de las problemáticas que enfrentan las organizaciones es la contratación de personal idóneo, esto es con las competencias requeridas para cada cargo; de allí que las universidades requieren que sus programas académicos respondan a las necesidades del entorno, al tiempo que se propende por una formación integral, no solo para el empleo, sino para la interrelación del sujeto en el mundo (Álvarez y De Prada, 2018). 
En este sentido, el presente trabajo analiza la percepción de la calidad de las prácticas realizadas por estudiantes de pregrado en Tecnología en Producción Industrial e Ingeniería Industrial, de una IES de la ciudad de Medellín, en la que interactúan tres poblaciones: universidad, empresas y practicantes, a partir de la medición de las competencias desarrolladas por estos últimos, mediante una mirada compleja del objeto de estudio, teniendo en cuenta competencias de distintos ámbitos, relaciones entre variables determinadas por los grupos de interés y las dimensiones de cada competencia, así como un análisis estadístico riguroso.

Frente al tema de estudio, en el ámbito nacional se encontraron algunos trabajos investigativos relacionados; sin embargo, en ninguno de ellos se evidenció el uso de herramientas estadísticas avanzadas, y tampoco se encontró alguno que analizara al tiempo la percepción de estudiantes, docentes y empresarios (ver los trabajos de Albert, García y Pérez, 2017; Álvarez, De Prada y Pino, 2018; Arias, García y Cardona, 2019; Cabrera, López y Portillo, 2016; Del Castillo, Nieto, Orozco, Pérez y Rubio, 2012; Martínez y González, 2018; Morelos y Vergara, 2011; Silva y Mazuera, 2019). En el contexto internacional se tienen algunos artículos de referencia, como los de Cayambe, Filian, Arguello y Rodríguez (2018); Chan, Mena, Escalante y Rodríguez (2018) o Herbert, Rothwell, Glover \& Lambert (2020), quienes trabajan el tema de las competencias en educación superior, la empleabilidad y el desempeño. Otras investigaciones como las de Chan, Fong, Luk \& Ho (2017); Badcock, Pattison \& Harris (2010); Domingo, Albajez \& Santolaria (2013); Guerrero, Palma \& La Rosa (2014); Kachalov et al. (2015); Shamshina (2014); Paton \& Wagner (2014) o Palma, Ríos \& Guerrero (2012) enfocan su análisis en los procesos de formación por competencias en educación superior.

En este trabajo, particularmente se pretende probar la hipótesis de que las percepciones de cada grupo, sobre las competencias del practicante, son iguales, a través del análisis de sus medias aritméticas y tomando como referencia los practicantes de las carreras antes mencionadas, en el semestre 2019-1. Para tal efecto, se hizo uso de un software estadístico (SPSS) y se utilizó como herramienta para la recolección de información primaria: la encuesta. En la primera parte del artículo se hace una revisión de literatura, en torno al concepto de competencias; posteriormente, se describe con detalle la metodología empleada, el instrumento de recolección de información y el análisis estadístico realizado, con sus respectivos resultados; luego se hace una discusión en torno a dichos resultados y se muestran las conclusiones del estudio. Se espera que esta investigación sirva de soporte a proyectos similares, teniendo en cuenta que, si bien el estudio toma un caso particular, la metodología, los referentes teóricos y el análisis estadístico pueden ser extrapolados en otros contextos, ajustando aspectos como población, muestra y competencias específicas a evaluar.

\section{| Referentes conceptuales}

El concepto de competencia tiene diferentes interpretaciones, según el contexto en que se analice, así como el paradigma dominante o escuela de pensamiento que aborde su estudio (Cuadra, Castro \& Juliá, 2018); una de sus definiciones habla de la capacidad de realizar una tarea de forma adecuada, aplicando conocimientos adquiridos en un proceso de formación; lo anterior, en contravía de la educación tradicional, basada en la transmisión de conocimientos, en tanto se pretende una formación integral, tanto en el Saber como en el Ser (Palmer, Montaño y Palou, 2009). Para Salcines, González, Ramírez y Martínez (2018), las competencias 
tienen que ver con el conocimiento, puesto en acción en forma de habilidades, valores y actitudes, y son esenciales para ejercer de forma adecuada una labor. Por su lado, para Tejada y Ruiz (2016) las competencias están constituidas por "conocimientos, procedimientos y actitudes" (p. 19), los cuales se integran en el ejercicio profesional; opinión que comparten Gómez, Lacasta, Martínez y Rodríguez (2018) cuando hablan de la naturaleza integradora de las competencias.

Neri y Hernández (2019), por su parte, ven en la competencia profesional una forma de asumir una posición específica en una organización, frente a un determinado evento, lo cual tiene que ver con la aplicación de conocimientos y rasgos de la personalidad; para los autores, una competencia está definida por un conjunto de saberes y actitudes que se ponen de manifiesto en situaciones reales, tanto laborales como personales. Para Montes de Oca y Machado (2014) las competencias son una consecuencia de la interacción entre personas y son desarrolladas a partir de procesos educativos y de la práctica constante en contextos reales; Cano (2008), por su parte, afirma que los diseños curriculares por competencias se justifican en tres hechos fundamentales: el avance de la sociedad del conocimiento, la complejidad como nuevo paradigma y la necesidad de una formación integral; de igual manera, ve en la evaluación por competencias una cuestión clave, para la cual se deben tener en cuenta aspectos como que la misma es a la vez causa y efecto del proceso de aprendizaje, además dicha evaluación no debe ser usada solo para seleccionar o categorizar las personas según sus habilidades y conocimientos, y debe ser coherente con todo el modelo educativo, para lo cual se requiere el uso de las herramientas y estrategias de enseñanza-aprendizaje adecuadas (Rico-Reintsch, 2019).

Autores como Fernández (2010) y Fernández y Duarte (2013) definen la competencia como una compleja estructura de atributos que determina la manera de actuar de un individuo, a partir de la movilización de habilidades cognitivas para afrontar situaciones de la vida diaria, tanto en lo personal como lo laboral. Por su parte, Valera (2010) define las competencias como una mezcla entre conocimientos, habilidades, comportamientos y actitudes, y enumera algunas cualidades de un profesional competente: capacidad para apropiarse de conceptos teóricos, habilidad en su desempeño, consciencia de la importancia de realizar bien su labor, ética profesional, destreza creativa e innovadora, autodidacta y capacidad de transferir el conocimiento. Kachalov et al. (2015) analizan la necesidad de las competencias interdisciplinarias en la educación superior, mientras que Saorín et al. (2017) hablan de la importancia del desarrollo de competencias, como la capacidad creativa en estudiantes de ingeniería.

De otro lado, Guerrero \& De los Ríos (2012) hablan de modelos de competencias profesionales, basados en aspectos como la estrategia empresarial, el conocimiento cognitivo y motivacional, el sitio de trabajo, la teoría del comportamiento y el enfoque holístico. García y Pérez (2008), entre tanto, mencionan el dilema que tienen las universidades para formar profesionales con las habilidades necesarias para ser competentes en una labor, entendiendo que la relación entre empleabilidad y valoración de competencias está determinada por el mercado laboral y el perfil de egreso del estudiante; de igual forma, los autores en mención, presentan un resumen (tabla 1) con algunas definiciones del concepto de competencia. 
Tabla 1

Definición de competencias

Referencia

Ministerio de Trabajo y Seguridad Social

OIT

DESECO (OCDE, 2002)

Mertens (1996)

Proyecto Tuning

Martínez, y Sauleda (2005)

\section{Definición de competencia}

La capacidad de aplicar conocimientos, habilidades y actitudes al desempeño de la ocupación de que se trate. Capacidad efectiva para llevar a cabo exitosamente una actividad laboral plenamente identificada.

Las habilidades para responder a las demandas a llevar a cabo tareas con éxito y consistentes con las dimensiones cognitivas y no cognitivas.

Capacidades de trabajo que están conformadas por una serie de conocimientos, actitudes y aptitudes, hábitos, valores y motivaciones dirigidas al desarrollo profesional.

Una combinación dinámica de atributos, en relación a conocimientos, habilidades, actitudes y responsabilidades, que describen los resultados de aprendizaje de un programa educativo o lo que los alumnos son capaces de demostrar al final de un proceso educativo.

Capacidades internas, destrezas, habilidades, dominio, prácticas o experticia alcanzada por el aprendiz, por lo que precisa de una enseñanza centrada en el estudiante y de una evaluación que no tenga solo en cuenta los conocimientos de los contenidos curriculares, sino del saber hacer procedimental. Es la capacidad de responder a demandas complejas y llevar a cabo tareas diversas de forma adecuada. Supone una combinación de habilidades prácticas, conocimientos, motivación, valores éticos, actitudes, emociones y otros componentes sociales y de comportamiento que se movilizan conjuntamente para lograr una acción eficaz.

Nota: Tomado de García y Pérez (2008)

También, Del Castillo et al. (2012) definen la competencia como la integración adecuada de acciones, destrezas, habilidades y prácticas, y las clasifican en metodológicas, técnicas, laborales, sociales, personales, interpersonales, de gestión y especificas; Jiménez, Hernández y González (2013), de igual manera, dividen las competencias en: técnicas, metodológicas, sociales y participativas. Larrain y González (2007), por su lado, entienden las competencias como un "saber con conciencia", a través del cual se pueda transformar la realidad, y solucionar problemáticas complejas, a partir del uso del conocimiento y valores adquiridos, mientras que, para Shamshina (2014), aspectos como la comunicación, la capacidad de resolución de problemas, la actitud hacia la mejora continua y el uso adecuado de los recursos son fundamentales para un ingeniero de este siglo. En este sentido, Paton \& Wagner (2014) realizan un 
estudio para determinar la relación entre la formación en competencias en gestión estratégica, en programas de ingeniería, y el desempeño de los graduados en cargos directivos de empresas alemanas, sacando como conclusión que las facultades de ingeniería en este país han excluido de sus planes de estudio asignaturas relacionadas con esta temática, lo que genera una preocupación dado que muchos cargos estratégicos en las empresas son ocupados por ingenieros.

\section{| La enseñanza por competencias y su relación con la práctica profesional}

Frente a esta temática, Arias, Portilla y Flórez (2007) afirman que la enseñanza por competencias debe fomentar el aprendizaje significativo, de manera que la pertinencia de los contenidos curriculares pueda ser validada en contextos reales, mientras que Zambrano (2014) habla del principio de aprendizaje en la práctica. Por otra parte, Cuadra et al. (2018) afirman que la relación entre academia y sector productivo no ha funcionado de forma efectiva, lo que ha generado una brecha entre las competencias adquiridas y las requeridas en la práctica profesional. Para Silva y Mazuera (2019) el concepto de competencia parte de una visión economicista, en donde lo que se busca es establecer indicadores de desempeño profesional. Ronquillo et al. (2019), por otro lado, analizan el impacto que ha tenido la globalización en los procesos de formación llevados a cabo en las universidades, enfocándolos hacia el desarrollo de capacidades para encadenar los conocimientos teóricos con el ejercicio profesional, mientras que Cayambe et al. (2018) entienden que las competencias profesionales constituyen un proceso que se desarrolla a lo largo de la vida y que están mediadas por la relación teoría-práctica.

Para Andrade (2008), el enfoque por competencias evalúa la compatibilidad, comparabilidad y competitividad de la educación superior, mientras que Rial (2007) y Hamid, Álvarez y Torres (2017) afirman que en la educación por competencias la evaluación se centra en determinar el nivel de respuesta de un estudiante, ante situaciones relacionadas con su profesión. Ronquillo et al. (2019), por su parte, definen la formación por competencias como un proceso constructivo, en el cual no solo importa la adquisición de conocimientos técnicos sobre un área en particular, sino que requiere de una profunda reflexión en torno al compromiso ético y moral de la práctica de una profesión específica; para Hernández, Alvarado y Luna (2015) las estrategias en educación superior en este siglo deberían estar enfocadas hacia la enseñanza por competencias. Por otro lado, Chan et al. (2017) mencionan que el desarrollo de competencias en educación superior no puede ser efectivo sí las practicas pedagógicas, los planes de estudio y los objetivos misionales de las universidades no están alineados, en tanto que, para Herbert et al. (2020) la preocupación se centra en el proceso de transición de los graduados al mundo laboral, dado que es alli donde se van a validar las competencias adquiridas.

Avanzando en la revisión, Tirado et al. (2007) aseguran que la formación por competencias implica el mejoramiento de la relación entre universidad - sociedad - empresa, mientras que para autores como Morelos y Vergara (2011) debe medirse el impacto de los egresados en el medio y su aporte al desarrollo social. Vera et al. (2019) hablan de la misión de la universidad de preparar profesionales con capacidad de aprender continuamente, lo cual requiere la 
adaptación de los sujetos para asumir el cambio y replantear el conocimiento adquirido; para los autores, un medidor importante de la calidad educativa se da en el desempeño profesional, idea que comparten Martínez y González (2018), Larrea y Alonso (2019), y Espinosa (2018), quienes ven como un reto para las universidades facilitar la inserción laboral y social de los profesionales, así como la pertinencia de sus programas; para Zúñiga, Leitón y Naranjo (2014), las situaciones de la vida diaria no están determinadas por un único campo disciplinar, por lo cual se requiere la movilización de habilidades cognitivas integrales que permitan una adecuada actuación en cada contexto, y esto es lo que define una competencia. Larrea y Alonso (2019), analizan la importancia de la evaluación de competencias profesionales, de manera que pueda asegurarse una formación integral.

Finalmente, Álvarez et al. (2018), así como Álvarez y De Prada (2018), plantean que la formación por competencias permite acercar la universidad a contextos reales, partiendo del supuesto que existe una brecha entre modelos educativos tradicionales y necesidades del entorno económico y social. Por último, entienden la importancia de las prácticas profesionales como una manera de acercar a los egresados al mundo real, a partir de su inserción en organizaciones donde pueden resolver problemáticas propias de sus áreas de estudio. Fernández (2010) afirma que la formación por competencias toma elementos de corrientes de pensamiento, como el constructivismo y el cognitivismo; concepción compartida por Villarroel y Bruna (2014), quienes agregan que la competencia permite la aplicación de conocimientos, habilidades, actitudes y valores para un adecuado desempeño en situaciones complejas. Ruiz, Gallardo, Navarro \& Cerón (2019) entienden que la formación por competencias requiere cambiar el paradigma de los modelos educativos basados en el docente, por modelos centrados en los estudiantes, a partir del aprendizaje colaborativo y basado en la resolución de problemas, mientras que Cristescu \& Draghicescu (2013) aportan a lo anterior cuando hablan del autoaprendizaje como una manera de fortalecer el desarrollo profesional.

\section{| Metodología}

El estudio se centró en comparar las competencias del Ser, del Saber y del Saber-Hacer en los practicantes de Tecnología en Producción Industrial e Ingeniería Industrial, de una IES de la ciudad de Medellín, a partir de la evaluación de tres grupos: estudiantes (32), coordinadores de práctica en las empresas (22) y docentes (34), durante el semestre académico 2019-1. Es preciso anotar que dichas muestras corresponden al total de estudiantes en práctica durante el semestre analizado y los docentes a cargo del proceso de formación en distintos niveles y asignaturas; dentro de los coordinadores, de un total de 32 empresas solo se tomaron los que respondieron de forma correcta toda la encuesta. También, se tomó como referencia, y para hacer comparativos, el informe realizado por la oficina de prácticas de la institución en el semestre 2019-1, con encuestas realizadas a 31 empresas y 35 practicantes, en la cual se indagaba acerca de la calidad y pertinencia de los programas. Se seleccionaron tres competencias con sus variables, con las que se elaboró un instrumento de recolección de información, mostrado en la tabla 2, el cual fue sometido a una prueba de validez de contenido. El alcance de este trabajo es del tipo comprensivo y con enfoque mixto. El diseño corresponde a una estrategia para obtención de información empírica del tipo no experimental, transeccional - correlacional. 
Tabla 2

Instrumento para recolección de información

\begin{tabular}{|c|c|c|}
\hline Dimensión & Variable & $\begin{array}{l}\text { Pregunta } \\
\end{array}$ \\
\hline \multirow{10}{*}{ SER } & Adaptabilidad & $\begin{array}{l}\text { ¿Tiene habilidad para desempeñarse con eficacia en cualquier } \\
\text { contexto? ¿Tiene capacidad de trabajar bajo presión? ¿Tiene } \\
\text { capacidad de trabajar en equipo y posee un buen manejo de las } \\
\text { relaciones interpersonales? }\end{array}$ \\
\hline & Entusiasmo & $\begin{array}{l}\text { ¿Es proactivo y cuenta con la disposición para realizar las } \\
\text { diferentes tareas que le asignen? ¿Realiza propuestas de mejora? }\end{array}$ \\
\hline & Flexibilidad & $\begin{array}{l}\text { ¿Tiene la capacidad para modificar positivamente la conducta } \\
\text { cuando surgen problemas? ¿Se adapta a los cambios generados } \\
\text { por la organización? }\end{array}$ \\
\hline & Impacto & $\begin{array}{l}\text { ¿Demuestra autoconfianza? ¿Es coherente con sus principios y } \\
\text { valores? ¿Demuestra respeto a sus superiores y compañeros? }\end{array}$ \\
\hline & $\begin{array}{l}\text { Poder de } \\
\text { recuperación }\end{array}$ & $\begin{array}{l}\text { ¿Supera los impases de la cotidianidad? ¿Cumplen con las tareas } \\
\text { y/o actividades eficazmente? }\end{array}$ \\
\hline & $\begin{array}{l}\text { Tolerancia a la } \\
\text { presión }\end{array}$ & $\begin{array}{l}\text { ¿Cumple con sus tareas adecuadamente? ¿Mantiene un buen } \\
\text { desempeño bajo presión? }\end{array}$ \\
\hline & Iniciativa & $\begin{array}{l}\text { ¿Posee iniciativa al mejoramiento, es innovador y creativo en los } \\
\text { procesos de la organización? ¿Es proactivo y productivo? }\end{array}$ \\
\hline & Liderazgo & $\begin{array}{l}\text { ¿Inspira y guía a los demás hacia la obtención de metas? } \\
\text { ¿Modifica su comportamiento para adaptarse a las tareas, } \\
\text { situaciones y personas? }\end{array}$ \\
\hline & $\begin{array}{l}\text { Sentido } \\
\text { urgencia }\end{array}$ & $\begin{array}{l}\text { ¿Percibe la urgencia de tareas y actúa para lograr su realización } \\
\text { en los plazos requeridos? ¿Sabe separar, clasificar y planear las } \\
\text { diferentes tareas según urgencias y prioridades? }\end{array}$ \\
\hline & Tenacidad & $\begin{array}{l}\text { ¿Es capaz de asimilar el cambio? ¿Cumple con los objetivos y } \\
\text { metas que fueron planteados por la organización? }\end{array}$ \\
\hline \multirow{6}{*}{ SABER } & $\begin{array}{l}\text { Sensibilidad } \\
\text { organizacional }\end{array}$ & $\begin{array}{l}\text { ¿Reconoce el efecto y los resultados de las propias decisiones en } \\
\text { otras partes de la organización? }\end{array}$ \\
\hline & Innovación & $\begin{array}{l}\text { ¿Propone soluciones creativas para resolver inconvenientes? } \\
\text { ¿Explora y evalúa diferentes maneras de enfrentar los problemas } \\
\text { y las oportunidades de la organización? }\end{array}$ \\
\hline & $\begin{array}{l}\text { Estándares de } \\
\text { trabajo }\end{array}$ & $\begin{array}{l}\text { ¿Es capaz de establecer herramientas de medición para el } \\
\text { desempeño adecuado en las labores que se le asignan? }\end{array}$ \\
\hline & $\begin{array}{l}\text { Atención al } \\
\text { detalle }\end{array}$ & $\begin{array}{l}\text { ¿Verifica con precisión los procesos y las tareas, y mantener la } \\
\text { vigilancia por un período de tiempo? }\end{array}$ \\
\hline & $\begin{array}{l}\text { Orientación al } \\
\text { logro }\end{array}$ & $\begin{array}{l}\text { ¿Actúa en función de realizar el trabajo de la mejor manera } \\
\text { posible o por sobrepasar los estándares establecidos? }\end{array}$ \\
\hline & Análisis & $\begin{array}{l}\text { ¿Relaciona y compara datos de diversas fuentes e identificar } \\
\text { relaciones de causa y efecto? }\end{array}$ \\
\hline $\begin{array}{l}\text { SABER } \\
\text { HACER }\end{array}$ & $\begin{array}{l}\text { Habilidad } \\
\text { numérica }\end{array}$ & $\begin{array}{l}\text { ¿Tiene la habilidad para analizar, organizar y presentar datos } \\
\text { numéricos, datos financieros y estadisticos? }\end{array}$ \\
\hline
\end{tabular}




\begin{tabular}{lll}
$\begin{array}{l}\text { Manejo } \\
\text { tecnología }\end{array}$ & de & $\begin{array}{l}\text { ¿Utiliza de forma óptima los equipos y máquinas requeridos en } \\
\text { procesos especificos en la empresa? }\end{array}$ \\
\hline $\begin{array}{l}\text { Planeación } \\
\text { organización }\end{array}$ & $\begin{array}{l}\text { ¿Planea adecuadamente requerimientos de personal y asignación } \\
\text { de recursos necesarios en procesos específicos de la empresa? }\end{array}$ \\
\hline Seguimiento & $\begin{array}{l}\text { ¿Establece procedimientos para monitorear los resultados de la } \\
\text { delegación, las asignaciones o los proyectos a cargo? }\end{array}$ \\
\hline $\begin{array}{l}\text { Toma } \\
\text { decisiones }\end{array}$ & de & $\begin{array}{l}\text { ¿Demuestra capacidad para la toma de decisiones de forma } \\
\text { analítica y acorde con las necesidades del contexto? }\end{array}$ \\
\hline $\begin{array}{l}\text { Comunicación } \\
\text { escrita }\end{array}$ & $\begin{array}{l}\text { ¿Expresa claramente sus ideas de manera escrita, con estructuras } \\
\text { gramaticales, léxico y terminología correctos? }\end{array}$ \\
\hline $\begin{array}{l}\text { Comunicación } \\
\text { oral }\end{array}$ & $\begin{array}{l}\text { ¿Expresa ideas de manera efectiva en situaciones individuales y } \\
\text { de grupo? }\end{array}$ \\
\hline $\begin{array}{l}\text { Trabajo } \\
\text { equipo }\end{array}$ & $\begin{array}{l}\text { ¿Cuenta con la habilidad para participar activamente de una meta } \\
\text { común? ¿Participa de forma activa y constructiva en los grupos } \\
\text { de trabajo? }\end{array}$ \\
\hline
\end{tabular}

Nota: elaboración propia.

Todos los îtems se valoran en formato Likert, así: 1 corresponde a "No cumple", 2 corresponde a "Cumple parcialmente", 3 corresponde a "Cumple" y 4 corresponde a "Cumple totalmente". Para comparar estadísticamente las medias del conjunto de competencias se clasificaron en las variables que puedan ser medibles, derivadas del marco teórico; la prueba estadística radica en probar una hipótesis alternativa que demuestre que al menos dos no son iguales.

\section{| Hipótesis}

$\mathrm{H}_{10}$ : Las medias de la competencia del Ser medidas en los tres grupos no son diferentes.

$\mathrm{H}_{11}$ : Las medias de la competencia del Ser medidas en los tres grupos son diferentes.

$\mathrm{H}_{20}$ : Las medias de la competencia del Saber medidas en los tres grupos no son diferentes.

$\mathrm{H}_{21}$ : Las medias de la competencia del Saber medidas en los tres grupos son diferentes.

$\mathrm{H}_{30}$ : Las medias de la competencia del Saber - Hacer medidas en los tres grupos no son diferentes.

$\mathrm{H}_{31}$ : Las medias de la competencia del Saber - Hacer medidas en los tres grupos son diferentes.

La prueba estadística a considerar inicialmente fue el Análisis de Varianza - ANOVA, una prueba paramétrica cuya potencia radica en la capacidad de comparar diferencias cuando estas existen (Walpole, R. Myers y S. Myers, 2012). El objetivo, por tanto, deriva de un conjunto de observaciones sobre $p$ variables, en una nueva variable constituida, usando una combinación lineal de p variables. Los pesos que definen esta combinación lineal se determinan para maximizar una estadística F, para dos o más grupos. En este caso, se han sumado las respuestas para cada una de las competencias (variables): Ser, Saber y Saber - Hacer, lo cual permite tener en cuenta la correlación entre variables. Las pruebas estadísticas univariadas, que se realizan en medias correlacionadas, no son independientes, de ahí la necesidad de una prueba con respecto a todas las variables. Por otro lado, se debe tener en cuenta que cada prueba univariante de tres poblaciones se construye de tal manera que permita un porcentaje de error $\alpha$, que será del $5 \%$, si la hipótesis nula se rechaza cuando es verdadera. En el ANOVA, la variación total se subdivide en variación que se debe a las diferencias entre los grupos y a la 
variación que obedece a las diferencias dentro de los grupos. Para realizar el análisis se consideró un nivel de significancia de $\alpha=0,05$.

Posteriormente, se realizaron las pruebas de normalidad de Shapiro-Wilk, homogeneidad de varianzas de Levene; finalmente, dado que no se cumplieron los supuestos para usar la ANOVA de un factor, se realizó la prueba de Kruskal - Wallis.

Es preciso aclarar que la variación dentro del grupo se considera un error aleatorio. La variación entre el grupo se debe a diferencias de un grupo a otro y también se conoce como efecto del tratamiento. Los tres grupos que se han considerado en estudio, representan poblaciones independientes, cuyos valores se han seleccionado en forma aleatoria, que deben seguir una distribución normal y además deben tener varianzas iguales para que se cumpla la hipótesis nula de no diferencia entre las medias de cada población.

Por último, se debe considerar que tanto la conceptualización teórica, la metodología empleada, así como la herramienta de obtención de información y los análisis estadísticos son susceptibles de ser utilizados en otros estudios, pero los resultados obtenidos, por este en particular, corresponden a una población y contextos específicos, de allí que no necesariamente reflejen una generalidad, en torno a la percepción de los procesos de formación por competencias en educación superior.

\section{| Resultados}

En la tabla 3 se muestra la comparación de mediciones en los grupos, y en la tabla 4 se presenta información que describe la composición de los parámetros para cada una de las tres muestras. 
Tabla 3

Tabla ANOVA para comparar las mediciones en los grupos

\begin{tabular}{|c|c|c|c|c|c|c|}
\hline \multicolumn{2}{|c|}{ Competencia } & Suma de & gl & Media & $\mathrm{F}$ & Sig \\
\hline \multirow{4}{*}{ Ser * Evaluador } & $\begin{array}{l}\text { Entre grupos } \\
\text { (Combinado) }\end{array}$ & 1611,021 & 2 & 805,510 & \multirow[t]{4}{*}{$\begin{array}{l}6,56 \\
2\end{array}$} & \multirow[t]{4}{*}{$\begin{array}{l}0,00 \\
2\end{array}$} \\
\hline & Dentro de grupos & 10433,29 & $\begin{array}{l}8 \\
5\end{array}$ & \multirow[t]{3}{*}{122,745} & & \\
\hline & \multirow{2}{*}{ Total } & 12044,31 & 8 & & & \\
\hline & & 8 & 7 & & & \\
\hline \multirow{3}{*}{ Saber * Evaluador } & $\begin{array}{l}\text { Entre grupos } \\
\text { (Combinado) }\end{array}$ & 116,245 & 2 & 58,123 & \multirow[t]{3}{*}{$\begin{array}{l}5,23 \\
5\end{array}$} & \multirow[t]{3}{*}{$\begin{array}{l}0,00 \\
7\end{array}$} \\
\hline & Dentro de grupos & 943,743 & $\begin{array}{l}8 \\
5\end{array}$ & \multirow[t]{2}{*}{11,103} & & \\
\hline & Total & 1059,989 & $\begin{array}{l}8 \\
7\end{array}$ & & & \\
\hline \multirow{3}{*}{$\begin{array}{l}\text { Saber Hacer } \\
\text { Evaluador }\end{array}$} & $\begin{array}{l}\text { Entre grupos } \\
\text { (Combinado) }\end{array}$ & 254,706 & 2 & 127,353 & \multirow[t]{3}{*}{$\begin{array}{l}2,52 \\
1\end{array}$} & \multirow[t]{3}{*}{$\begin{array}{l}0,08 \\
6\end{array}$} \\
\hline & Dentro de grupos & 4293,192 & $\begin{array}{l}8 \\
5\end{array}$ & \multirow[t]{2}{*}{50,508} & & \\
\hline & Total & 4547,898 & $\begin{array}{l}8 \\
7\end{array}$ & & & \\
\hline
\end{tabular}

Nota: Elaboración propia basados en SPSS

Tabla 4

Información descriptiva de las muestras

\begin{tabular}{|c|c|c|c|c|c|c|c|c|}
\hline \multirow{2}{*}{ Evaluador } & \multicolumn{2}{|c|}{ Docente $\left(\mathrm{N}_{\mathrm{D}}=34\right)$} & \multicolumn{2}{|c|}{ Alumno $\left(\mathrm{N}_{\mathrm{A}}=32\right)$} & \multicolumn{2}{|c|}{$\begin{array}{l}\text { Coordinador } \\
\left(\mathrm{N}_{\mathrm{C}}=22\right)\end{array}$} & \multicolumn{2}{|c|}{ Total $\left(\mathrm{N}_{\mathrm{T}}=88\right)$} \\
\hline & Media & $\begin{array}{l}\text { Desv. } \\
\text { estándar }\end{array}$ & Media & $\begin{array}{l}\text { Desv. } \\
\text { Estándar }\end{array}$ & Media & $\begin{array}{l}\text { Desv. } \\
\text { estándar }\end{array}$ & Media & $\begin{array}{l}\text { Desv. } \\
\text { estándar }\end{array}$ \\
\hline Ser & 81,21 & 11,286 & 91,06 & 9,301 & 85,23 & 12,998 & 85,8 & 11,766 \\
\hline $\begin{array}{l}\text { Saber } \\
\text { Saber } \\
\text { Hacer }\end{array}$ & $\begin{array}{l}20,97 \\
45,94\end{array}$ & $\begin{array}{l}3,555 \\
6,924\end{array}$ & $\begin{array}{l}23,62 \\
49,84\end{array}$ & $\begin{array}{l}2,927 \\
6,687\end{array}$ & $\begin{array}{l}22,18 \\
47,36\end{array}$ & $\begin{array}{l}3,527 \\
7,944\end{array}$ & $\begin{array}{l}22,24 \\
47,72\end{array}$ & $\begin{array}{l}3,491 \\
7,23\end{array}$ \\
\hline
\end{tabular}

Nota: Elaboración propia basados en SPSS 
Antes de evaluar la prueba se debe verificar sí se cumplen los supuestos para usar la prueba F con ANOVA, acerca de los datos: aleatoriedad e independencia, normalidad, homogeneidad de varianzas. En este caso, se dividió la muestra en grupos; cada grupo corresponde a una población diferente. Las desviaciones de este supuesto pueden afectar las inferencias del análisis de varianza. El supuesto de normalidad establece que los valores de la muestra en cada grupo provienen de una población distribuida normalmente.

\section{| Exploración de Pruebas de Normalidad}

Se desea comparar las evaluaciones realizadas por los 3 grupos, mediante procedimientos paramétricos. Para realizar el análisis se ha cambiado la escala de las variables, con el fin de hacer comparables las medias, tomando el valor de la competencia, restándole la media y dividiendo por el valor estándar de la media. La tabla 5 contiene información descriptiva que permite analizar la información al respecto.

Tabla 5

Descriptivos de Normalidad de las variables aleatorias competencias

\begin{tabular}{|c|c|c|c|c|}
\hline \multicolumn{5}{|l|}{ Competencia del ser } \\
\hline \multicolumn{2}{|l|}{ Rol } & Docente & Alumn & Coordinado \\
\hline \multicolumn{2}{|l|}{ Media } & $-0,39$ & 0,45 & $-0,05$ \\
\hline \multicolumn{2}{|l|}{ Error estándar de la media } & 0,16 & 0,14 & 0,24 \\
\hline \multirow{2}{*}{$\begin{array}{l}95 \% \text { de intervalo de confianza para la } \\
\text { media }\end{array}$} & Limite & $-0,71$ & 0,17 & $-0,51$ \\
\hline & $\begin{array}{l}\text { Limite } \\
\text { superior }\end{array}$ & $-0,07$ & 0,72 & 0,41 \\
\hline \multicolumn{5}{|l|}{ Competencia del saber } \\
\hline \multirow{4}{*}{$\begin{array}{l}\text { Media } \\
\text { Error estándar de la media } \\
95 \% \text { de intervalo de confianza para la } \\
\text { media }\end{array}$} & & $-0,36$ & 0,40 & $-0,01$ \\
\hline & & 0,17 & 0,15 & 0,22 \\
\hline & $\begin{array}{l}\text { Limite } \\
\text { inferior }\end{array}$ & $-0,70$ & 0,11 & $-0,44$ \\
\hline & $\begin{array}{l}\text { Limite } \\
\text { superior }\end{array}$ & $-0,02$ & 0,69 & 0,41 \\
\hline \multicolumn{5}{|l|}{ Competencia del Saber-Hacer } \\
\hline \multirow{4}{*}{$\begin{array}{l}\text { Media } \\
\text { Error estándar de la media } \\
95 \% \text { de intervalo de confianza para la } \\
\text { media }\end{array}$} & & $-0,25$ & 0,29 & $-0,05$ \\
\hline & & 0,16 & 0,16 & 0,23 \\
\hline & $\begin{array}{l}\text { Limite } \\
\text { inferior }\end{array}$ & $-0,57$ & $-0,03$ & $-0,51$ \\
\hline & $\begin{array}{l}\text { Limite } \\
\text { superior }\end{array}$ & 0,08 & 0,61 & 0,41 \\
\hline
\end{tabular}


Como se puede notar en algunos casos, el valor de la media de una competencia no está contenido en los intervalos de confianza de la media de las otras evaluaciones. Por este motivo, se puede concluir que las medias no son iguales, si y solo si las distribuciones de las competencias tienen distribución normal. De igual manera, se evidencia una diferencia significativa entre las medias de cada población en cada competencia, lo cual no solo podría afirmar lo enunciado anteriormente, sino que además mostraría una marcada diferencia de percepciones frente a las competencias desarrolladas.

\section{| Planteamiento de hipótesis}

Para demostrar sí las variables tienen una distribución normal se realizó una prueba de normalidad, con un nivel de significancia de $\alpha=0,05$.

$\mathrm{H}_{0}$ : La distribución de la variable aleatoria no es diferente a una distribución normal

$\mathrm{H}_{1}$ : La distribución de la variable aleatoria es diferente a una distribución normal. La prueba usada es la Shapiro - Wilk (tabla 6), la cual se ajusta al tamaño de la muestra (menor a 50 observaciones por grupo); para valores de $p>0,05$ se acepta la hipótesis nula; esto es, la distribución de variables aleatorias no es diferente a una distribución normal.

Tabla 6

Prueba de Normalidad de Shapiro-Wilk

\begin{tabular}{llllll}
\hline \multicolumn{1}{l}{ Prueba de normalidad } & \multicolumn{5}{l}{} \\
& Evaluador & \multicolumn{2}{l}{ Shapiro-Wilk } & \\
Ser & Estadistico & gl & Sig. & Decisión \\
& Docente & 0,926 & 34 & 0,024 & Se rechaza $\mathrm{H}_{0}$ \\
& Alumno & 0,930 & 32 & $\mathbf{0 , 0 4 0}$ & Se rechaza $\mathrm{H}_{0}$ \\
Saber & Coordinador Práctica & 0,944 & 22 & 0,244 & Se acepta $\mathrm{H}_{0}$ \\
& Docente & 0,923 & 34 & 0,019 & Se rechaza $\mathrm{H}_{0}$ \\
& Alumno & 0,950 & 32 & 0,142 & Se acepta $\mathrm{H}_{0}$ \\
Saber Hacer & Coordinador Práctica & 0,884 & 22 & 0,015 & Se rechaza $\mathrm{H}_{0}$ \\
& Docente & 0,951 & 34 & 0,136 & Se acepta $\mathrm{H}_{0}$ \\
& Alumno & 0,961 & 32 & 0,295 & Se acepta $\mathrm{H}_{0}$ \\
& Coordinador Práctica & 0,911 & 22 & 0,050 & Se acepta $\mathrm{H}_{0}$ \\
\hline
\end{tabular}

\section{Nota: Elaboración propia basada en SPSS}

Se puede concluir que la única variable que corresponde en todos los grupos a una distribución normal es la competencia del Saber - Hacer. No se pueden comparar las variables, por tal motivo no puede ser usada la técnica de Análisis de Varianza de una vía. Lo anterior, se puede explicar a partir del hecho de que la IES objeto de estudio tiene una amplia tradición en carreras tecnológicas, de allí que sus procesos formativos están más enfocados en el Saber Hacer, en detrimento de las competencias del Ser y el Saber. 


\section{| Exploración de Prueba de Homogeneidad de Varianzas}

La prueba estadística $\mathrm{F}$ de la ANOVA es comparativamente robusta, en relación con los supuestos de las varianzas de grupos iguales; la existencia de enormes diferencias en las varianzas afectará el nivel de significancia y el poder de la prueba F (Walpole et al., 2012).

\section{| Planteamiento de hipótesis}

$\mathrm{H}_{0}$ : No existen diferencias significativas entre las varianzas en los tres grupos, es decir $\sigma$ ${ }^{2}$ Docentes $=\sigma^{2}$ Alumnos $=\sigma^{2}$ Coordinadores

$\mathrm{H}_{1}$ : Existen diferencias significativas entre las varianzas de al menos dos grupos. El nivel de significancia elegido es $\alpha=0,05$. Para valores de $p<0,05$ se rechaza la hipótesis nula.

La prueba Levene permite probar la igualdad de varianzas de poblaciones (tabla 7):

\section{Tabla 7}

\section{Prueba de homogeneidad de varianzas}

\begin{tabular}{|c|c|c|c|c|}
\hline Competencias & Estadistico de Levene & dfl & df2 & Sig. \\
\hline Ser & 0,960 & 2 & 85 & 0,387 \\
\hline Saber & 0,270 & 2 & 85 & 0,764 \\
\hline Saber Hacer & 0,675 & 2 & 85 & 0,512 \\
\hline
\end{tabular}

\section{Nola: Elaboración propia usando SPSS}

Con una significancia del $5 \%$, la prueba Levene si $p<0,05$ rechaza la hipótesis nula. Para la competencia del ser, el valor de $p=0,387>0,05$ (se acepta la hipótesis nula); para la competencia del saber, el valor de $p=0,764>0,05$ (no se rechaza la hipótesis nula); para la competencia del saber - hacer, el valor de $p=0,512>0,05$ (no se rechaza la hipótesis nula). No existe evidencia de una diferencia significativa entre las tres varianzas, por tal motivo no existen argumentos para concluir que las varianzas de las variables no sean homogéneas. Dado que no se cumplen los supuestos para usar la ANOVA de un factor se debe usar la ANOVA de Kruskal - Wallis.

Hipótesis:

$\mathrm{H}_{0}$ : la medición de las competencias no es distinta según el tipo de evaluador.

$\mathrm{H}_{1}$ : la medición de las competencias es distinta según el tipo de evaluador. El nivel de significancia es de $\alpha=0,05$. Para valores de $p<0,05$, se rechaza la hipótesis nula.

Para la medición de la competencia del Ser se concluye que las mediciones son distintas según el tipo de evaluador, ya que $p=0,003<\alpha=0,05$ (se rechaza la hipótesis nula). Para la medición de la competencia del Saber se concluye que las mediciones son distintas según el tipo de evaluador, ya que $p=0,011<\alpha=0,05$ (se rechaza la hipótesis nula). Para la medición de la competencia del Saber - Hacer se concluye que las mediciones no son distintas según el tipo de evaluador, ya que $p=0,102>\alpha=0,05$ (se acepta la hipótesis nula). La tabla muestra 8 que se rechaza $\mathrm{HO}$, es decir, que las percepciones de los evaluadores son distintas. 


\section{Tabla 8}

Prueba de Kruskal Wallis

\begin{tabular}{llll}
\hline \multicolumn{4}{c}{ Estadísticos de prueba $^{\mathbf{a}, \mathbf{b}}$} \\
\hline & Ser & Saber & Saber Hacer \\
\hline Chi-cuadrado & 11,902 & 9,049 & 4,572 \\
Grados de libertad & 2 & 2 & 2 \\
Sig. asintótica & 0,003 & 0,011 & 0,102 \\
a. Prueba de Kruskal Wallis & & \\
b. Variable de agrupación: tipo de evaluador & & \\
\hline
\end{tabular}

\section{Nota: elaboración propia, usando SPSS}

Esto implica que, para los grupos analizados, la percepción frente a las competencias adquiridas en el proceso formativo es distinta, lo cual a su vez supone una diferencia entre las expectativas y la realidad reflejada en el desempeño del practicante en la empresa. La visión de cada grupo frente a dicho proceso muestra cómo el desarrollo de una competencia requiere de una puesta en común entre las necesidades de las organizaciones, los objetivos de formación de las IES, y las expectativas académicas, personales y profesionales de los estudiantes.

\section{| Discusión}

La formación integral en un modelo por competencias implica grandes retos; la pertinencia de los programas académicos depende, en gran medida, de su capacidad para ajustar los contenidos temáticos a la realidad y las necesidades del contexto en que se van a desenvolver los futuros profesionales. Valera (2010) lo menciona cuando habla de una formación trascendente y flexible, ajustada a diseños curriculares por competencias. Ronquillo et al. (2019) entienden que, en un mundo en constante cambio, las políticas educativas deben ajustarse a nuevas realidades y exigencias. En esta misma línea, Badcock et al. (2010) mencionan la problemática que enfrentan las universidades para integrar, en sus planes de estudio, competencias, habilidades y destrezas para mejorar el desempeño en el mundo laboral, dado que aún muchas se encuentran inmersas en un paradigma de formación por contenidos, entendiendo de esta manera que no solo se trata de formar en el Saber, sino también en el Ser y el Saber- Hacer.

Trabajos relacionados con lo presentado en este artículo, tales como el de Arias, García et al. (2019), plantean un análisis de la apropiación de competencias en investigación formativa, a partir de las practicas realizadas por estudiantes de Microbiología de la Universidad de Antioquia, dejando como resultado el hecho de validar el impacto positivo de las prácticas en el proceso de formación. Un texto anterior, de Neri y Hernández (2019), hace un estudio sobre la percepción de estudiantes de ingeniería, en torno a las competencias que se desarrollan en sus programas académicos, y cuáles de dichas competencias son fundamentales para su ejercicio profesional. Por otro lado, Álvarez et al. (2018) analizan la correspondencia entre los procesos 
de formación y las necesidades del mercado, pero haciendo énfasis en la parte creativa, la cual obtiene una menor valoración dentro del estudio. Para Guerrero et al. (2014) son tan importantes las habilidades duras, relacionadas con el conocimiento técnico de la profesión, como las blandas, las cuales facilitan aspectos como la comunicación asertiva, el trabajo en economía o el aprendizaje continuo.

De otro lado, el trabajo de Del Castillo et al. (2012) se enfoca específicamente en la descripción de los criterios de evaluación de competencias, en las prácticas realizadas por estudiantes de Ingeniería Industrial de la Universidad Libre de Barranquilla, y presenta como principal conclusión una evaluación satisfactoria de las competencias adquiridas. Martínez y González (2018) evalúan un instrumento que permite la adecuación a los requerimientos del mercado, en términos de competencias a partir de un análisis psicométrico; así mismo, validan los resultados obtenidos con un modelo estadístico de ecuaciones estructurales. Tirado et al. (2007), por su parte, desarrollan un estudio enfocado específicamente en las competencias profesionales de un ingeniero industrial, resaltando la importancia de los diseños curriculares por competencia; y Morelos y Vergara (2018) llevan a cabo una evaluación del impacto de los egresados de Ingeniería Industrial, en la ciudad de Barranquilla, haciendo énfasis en el concepto de calidad en la educación y postulando, además, como factores de impacto del egresado: su ámbito de incidencia, la influencia sobre el desarrollo social y su participación en contextos académicos, laborales y científicos.

Por su parte, Gallardo, López y Carter (2018) realizan un estudio para analizar la percepción de estudiantes de licenciaturas sobre las competencias adquiridas en sus carreras. También, Chisvert, Palomares, Hernaiz y Salinas (2018) presentan un análisis acerca del aprendizaje adquirido de las personas en empresas de inserción, y la manera como aprenden, entendiendo que dicho aprendizaje esta mediado por el contexto. Estudios similares al anterior son los desarrollados por Chan et al. (2018); Martínez, N. González y C. González (2016), y Jiménez, Gutiérrez y Hernández (2019), en donde evalúan la satisfacción de los estudiantes en torno a las competencias adquiridas. Cabrera et al. (2016) enfocan su investigación en el análisis de la percepción de las empresas sobre las competencias que requiere un profesional para ser exitoso; además, concluyen en su estudio que los empleadores dan una mayor importancia a las competencias genéricas, tales como el trabajo en equipo o el liderazgo, que a las competencias técnicas. Una investigación muy similar a la presentada en este documento es la planteada por Albert et al. (2017), quienes toman tres grupos de interés para hacer una valoración de las necesidades formativas y la empleabilidad en un programa de posgrado en ingeniería.

El presente trabajo aporta a la discusión sobre la relación entre los procesos de formación por competencias y las prácticas profesionales, dado que el análisis desarrollado se hace tomando como referencia los directos implicados en dicho proceso (estudiantes, docentes, empresas, universidad), lo cual permite una visión más amplia del fenómeno estudiado. Así las cosas, un análisis riguroso no debe estar orientado a la validación de un modelo educativo o de un proceso de enseñanza-aprendizaje en particular, sino que debe permitir el debate en torno a las fortalezas y debilidades de estos, de manera que se puedan establecer planes de mejoramiento acordes con el contexto. En este sentido, con la presente investigación se obtiene un insumo importante para mejorar los procesos educativos en instituciones de educación superior, y la aplicabilidad del estudio radica en la potencia y rigurosidad del análisis estadístico empleado, además de la pertinencia del objeto de investigación, dado la 
importancia que tiene para las IES la evaluación adecuada de sus procesos de formación.

Frente a los resultados derivados de las pruebas estadísticas realizadas, se evidenció que las percepciones de los grupos de interés son distintas; las explicaciones que se pueden plantear podrian ser diversas, partiendo del hecho de que los grupos en cuestión tienen diferentes expectativas frente al proceso de formación, lo cual a su vez genera la inquietud acerca de la manera adecuada de direccionar dicho proceso, de forma que esté acorde con los requerimientos y necesidades de los distintos implicados.

Partiendo del concepto teórico de competencia, analizado a lo largo de este texto, se podría pensar que la divergencia entre las percepciones resalta el hecho de que al tratarse de un proceso constante, cambiante y en ocasiones subjetivo, la formación no responde solamente a un desarrollo de contenidos curriculares, sino que depende de otros factores que presentan cierta dificultad para su medición, de allí que se hable más de percepción que de un indicador numérico preciso para validar dicho proceso. Sin embargo, los resultados sí pueden mostrar ciertas tendencias, que pueden ser tratadas como factores de análisis en posteriores procesos de mejoramiento. Por ejemplo, se evidencia cómo la evaluación de competencias está sujeta no solo a la parte técnica, sino también a la necesidad de medir factores intangibles, muy importantes para el desempeño profesional, relacionados con las competencias del Ser; Domingo et al. (2013), aportan en este sentido cuando hablan de las competencias genéricas y específicas, así como Palma et al. (2012), quienes expresan que los programas de ingeniería no solo deben contemplar competencias especializadas de conocimiento, sino que las empresas y la sociedad urgen de habilidades básicas para afrontar el cambio.

Si se hace un comparativo con los resultados arrojados en la encuesta que realiza la oficina de prácticas de la institución, la cual indaga sobre la percepción en temas de calidad y pertinencia de los programas académicos, se tienen observaciones interesantes, que a su vez contrastan con las obtenidas en este trabajo. Por ejemplo, es evidente la necesidad de los programas en el medio, dado que para el semestre en estudio (2019-1) se tuvieron en total 120 solicitudes de practicantes (33 para Ingeniería Industrial y 87 para Tecnología en Producción Industrial), de las cuales solo se pudieron suplir 72 (31 en ingeniería y 41 en tecnología), en un total de 31 empresas. Frente al tema de los aspectos a mejorar en la parte académica, las empresas encuestadas resaltaron la importancia de fortalecer competencias relacionadas con el pensamiento numérico y complejo, el manejo de herramientas tecnológicas, y las de liderazgo y toma de decisiones, las cuales consideran como las más relevantes, opinión que confirman los estudiantes encuestados, quienes afirman que los programas son pertinentes, pero que se debe reforzar en los aspectos antes señalados.

Haciendo énfasis en los resultados obtenidos en este estudio, se tiene que los empresarios dan mayor importancia a competencias como la adaptabilidad, tolerancia a la presión, el liderazgo y toma de decisiones, el pensamiento innovador, la habilidad numérica y el trabajo en equipo; por su parte, los estudiantes muestran un notable desempeño en competencias relacionadas con el Saber- Hacer, lo cual es una fortaleza de la institución, pero tienen una menor valoración a las competencias del Ser y el Saber, lo cual sugiere que sería un tema por mejorar en los programas académicos. 


\section{| Conclusiones}

Cabe resaltar la importancia que se da en las organizaciones -independiente del sector económico al cual pertenecen- a la formación por competencias, dado que en muchos casos no está alineada con los planes de formación en las instituciones de educación superior, en las cuales aún se plantean modelos educativos de tipo transmisionistas, poco flexibles y alejados de la realidad del entorno. El aprendizaje significativo requiere de esquemas formativos que se enfoquen no solo en la adquisición de conocimientos teóricos, sino también en la aplicabilidad de los mismos para la solución de problemas en contextos específicos, tanto laborales como personales; de allí la importancia de medir el impacto de las prácticas empresariales, a partir de la percepción de los actores implicados (estudiantes, universidad, empresa), frente al proceso formativo y su pertinencia en el medio.

La percepción frente a una competencia presenta variaciones según el enfoque de cada evaluador; en este sentido, es importante analizar las expectativas de los distintos grupos de interés y las necesidades que plantea el sector industrial, frente a los procesos de formación en Ingeniería y Tecnología Industrial; lo anterior, plantea la posibilidad de llevar a cabo planes de actualización y mejoramiento de los contenidos curriculares y las estrategias de enseñanza aprendizaje dentro de dichos programas.

Frente a las resultados obtenidos a partir de la evaluación de la percepción de los tres tipos de competencias analizadas en los grupos de interés, se tiene como conclusión general lo siguiente: las competencias del Saber - Hacer son las mejor valoradas, lo cual es coherente con el modelo formativo de la IES en cuestión, y su larga trayectoria en formación a nivel tecnológico; en cuanto a las competencias del Saber, es claro que las principales falencias son relativas al área de ciencias exactas y aplicadas, y a conocimientos muy específicos como el manejo de herramientas tecnológicas y de ofimáticas, lo cual muestra que los planes de estudio deberían reforzarse en estas áreas; para las competencias del Ser, se tiene que son las menos trabajadas en la IES y son muy pertinentes, sobre todo para los empresarios quienes piden que los egresados desarrollen competencias blandas que les permitan interactuar en el entorno de forma asertiva, asumir posiciones de liderazgo y ser más propositivos. Se podría afirmar, con lo anterior, que un modelo de formación integral debe propender por el ofrecimiento, a los futuros profesionales, de herramientas no solo desde lo técnico, lo cual está directamente relacionado con su labor específica, sino que además debe velar por la formación de profesionales para el mundo, capaces de ofrecer soluciones creativas e innovadoras a las problemáticas de las empresas y de interactuar de forma adecuada en sus entornos laborales y sociales.

En general, los estudios sobre temas similares al de este artículo, realizados en algunas IES de Colombia, apuntan a medir aspectos como: pertinencia de los programas, calidad, fortalezas y debilidades, impacto de planes de mejoramiento y relación con el entorno; en este sentido, un trabajo investigativo como el presente puede servir como base para el desarrollo de otras investigaciones al respecto, que permitan a su vez obtener información relevante acerca de los procesos de formación por competencias y el impacto de los egresados en la industria.

Aunque pueden existir distintas definiciones del concepto de competencia, se podría decir que las mismas confluyen en torno a la capacidad de actuar de un individuo frente a 
situaciones específicas, tanto laborales como personales, haciendo uso del conocimiento adquirido y valores obtenidos dentro de un proceso de formación académico y personal. Es preciso anotar que frente al enfoque de formación por competencias se presentan algunas críticas referidas a aspectos como la falta de una concepción clara desde la teoría pedagógica y el hecho de surgir como una posible respuesta a la problemática empresarial en temas de capital humano.

Los resultados finales obtenidos plantean una posible ruta metodológica para la realización de proyectos de investigación en torno a la temática tratada; tanto el instrumento de recolección de información como los estadísticos de prueba empleados presentan un modelo de análisis viable para determinar, de forma adecuada, la percepción de grupos de interés frente a los procesos de formación por competencias, aplicados en un contexto especifico.

\section{| Referencias}

Albert, M., García, M. y Pérez, C. (2017). Competencias, Formación y Empleo. Análisis de Necesidades en un Programa de Master en Ingeniería. Formación Universitaria, 10(2), 43-56.

https://www.doi.org/10.4067/S0718-50062017000200006

Álvarez, M. y De Prada, E. (2018). Evaluación de las competencias profesionales a través de las prácticas externas: incidencia de la creatividad. Revista de Investigación Educativa, 36(1), 203-219. https://www.doi.org/10.6018/rie.36.1.275651

Álvarez, M., De Prada, E. y Pino, M. (2018). Estudio sobre la capacidad técnica y las competencias transversales desarrolladas en las prácticas externas universitarias. Revista Estudios Pedagógicos, 44(3), 137-155. https://www.doi.org/10.4067/S0718-07052018000300137

Andrade, R. (2008). El enfoque por competencias en educación. Ide@s CONCYTEG, 3(38), 53-64.

Arias, L., García, G. y Cardona, J. (2019). Impacto de las prácticas profesionales sobre las competencias de investigación formativa en estudiantes de Microbiología de la Universidad de Antioquia-Colombia. Revista Virtual Universidad Católica del Norte, (56), 2-15.

Arias, L., Portilla, L. y Flórez, M. (2007). Competencias y empleabilidad. Scientia et Technica, 13(37), 379-392. https://www.doi.org/10.22517/23447214.4125

Badcock, P., Pattison, P. \& Harris, K. (2010). Developing generic skills through university study: a study of arts, science and engineering in Australia. Higher Education, 60(4), 441-458. https://www.doi.org/10.1007/s10734-010-9308-8

Cabrera, N., López, M. y Portillo, M. (2016). Las competencias de los graduados y su evaluación desde la perspectiva de los empleadores. Estudios Pedagógicos, 42(3) 69-87. https://www.doi.org/10.4067//S0718-07052016000400004

Cano, M. (2008). La evaluación por competencias en la educación superior. Profesorado, 12(3), $1-16$. 


\section{Percepción del proceso | Revista virtual}

Cayambe, M., Filian., F., Arguello, L. y Rodríguez, N. (2018). Formación por competencias: reto actual de la universidad ecuatoriana. Didasc@lia: Didáctica y Educación, 9(1), 141-150.

Chan, C., Fong, E., Luk, L. \& Ho, R. (2017). A review of literature on challenges in the development and implementation of generic competencies in higher education curriculum. International Journal of Educational Development, 57, 1-10. https://www.doi.org/10.1016/j.ijedudev.2017.08.010

Chan, M., Mena, D., Escalante, J. y Rodríguez, M. (2018). Contribución de las Prácticas Profesionales en la formación de los Estudiantes de la Facultad de Ingeniería Química de la Universidad Autónoma de Yucatán (México). Formación Universitaria, 11(1), 53-62. https://www.doi.or$\mathrm{g} / 10.4067 /$ S0718-50062018000100053

Chisvert, M., Palomares, D., Hernaiz, N. y Salinas, M. (2018). Trayectorias de aprendizaje en contextos informales. El caso de tres empresas de inserción. CIRIEC-España, Revista de Economía Pública, Social y Cooperativa, (94), 185-216. https://www.doi.org/10.7203/ClRIEC-E.94.12695

Cristescu, M. \& Draghicescu, L. (2013). The formation of self-education and professional development competences at the students from the departments with technological profile. Procedia - Social and Behavioral Sciences, 76, 744-748. https://www.doi.org/10.1016/j.sbspro.2013.04.198

Cuadra, D., Castro, P. \& Juliá, M. (2018). Three Types of Knowledge in Competency Based Professional Education: The Integration of Subjective, Professional, and Scientific Theories. Formación universitaria, 11(5), 19-30. https://www.doi.org/10.4067/S0718-50062018000500019

Del Castillo, M., Nieto, K., Orozco, J., Pérez, G. y Rubio, M. (2012). Descripción de los criterios de evaluaciones de las competencias en el proceso de prácticas de los estudiantes de Ingeniería Industrial de la Universidad Libre Seccional Barranquilla. Ingeniare, 7(12), 47-61. https://www.doi.org/10.18041/1909-2458/ingeniare.12.634

Domingo, M., Albajez, J. \& Santolaria, J. (2013). The accreditation of industrial engineering in Spain: teaching and learning the skills of manufacturing engineering. Procedia Engineering, 63, 786-795. https://www.doi.org/10.1016/j.proeng.2013.08.174

Espinosa, M. (2018). Tendencias recientes de la vinculación universitaria con el entorno. Desafíos relacionados con una pertinencia social integral. Revista Iberoamericana de Educación Superior, 9(26), 110-129.

Fernández, A. (2010). La evaluación orientada al aprendizaje en un modelo de formación por competencias en la educación universitaria. Revista de Docencia Universitaria, 8(1), 11-34.

Fernández, F. y Duarte, J. (2013). El Aprendizaje Basado en Problemas como estrategia para el desarrollo de competencias específicas en estudiantes de ingeniería. Revista Formación Universitaria, 6(5), 29-38. https://www.doi.org/10.4067/S0718-50062013000500005

Gallardo, F., López, V. y Carter, B. (2018). Efectos de la aplicación de un sistema de evaluación formativa en la autopercepción de competencias adquiridas en formación inicial del profesorado. Estudios Pedagógicos, 44(2), 55-77. 
García, J. y Pérez, M. (2008). Espacio Europeo de Educación Superior: competencias profesionales y empleabilidad. Revista Iberoamericana de Educación, (46), 1-12.

Gómez, F., Lacasta, J., Martínez, V. y Rodríguez, C. (2018). Avances en el liderazgo: marco de competencias de los líderes profesionales. Siglo Cero, 49(4), 7-34. https://www.doi.or$\mathrm{g} / 10.14201 /$ scero2018494734

Guerrero, D. \& De los Ríos, I. (2012). Professional competences: a classification of international models. Procedia - Social and Behavioral Sciences, 46, 1290-1296. https://www.doi.org/10.1016/j.sbspro.2012.05.290

Guerrero, D., Palma, M. \& La Rosa, G. (2014). Developing competences in engineering students. The case of project management course. Procedia - Social and Behavioral Sciences, 112, 832-841. https://www.doi.org/10.1016/j.sbspro.2014.01.1239

Hamid, N., Álvarez, J. y Torres, M. (2017). Propuesta metodológica para medir la pertinencia y el impacto de programas de pregrado en ingeniería. Revista Educación en Ingeniería, 12(24), 97-100. https://www.doi.org/10.26507/ rei.v12n24.807

Herbert, I., Rothwell, A., Glover, J. \& Lambert, S. (2020). Graduate employability, employment prospects and workreadiness in the changing field of professional work. The International Journal of Management Education, 18, 1-13. https://www.doi.org/10.1016/j.ijme.2020.100378

Hernández, I., Alvarado, J. y Luna, S. (2015). Creatividad e innovación: competencias genéricas o transversales en la formación profesional. Revista Virtual Universidad Católica del Norte, (44), 135-151.

Jiménez, Y., Gutiérrez, J. y Hernández, J. (2019). Logros y Desafíos en la Formación de Competencias Transversales por áreas de Conocimiento en la Educación Superior del Instituto Politécnico Nacional (México). Formación Universitaria, 12(3), 91-100. https://www.doi.or$\mathrm{g} / 10.4067 /$ S0718-50062019000300091

Jiménez, Y., Hernández, J. y González, M. (2013). Competencias profesionales en la educación superior: justificación, evaluación y análisis. Innovación educativa, 13(61), 45-65.

Kachalov, N., Kornienko, A., Kvesko, R., Kornienko, A., Kvesko, S. \& Chaplinskaya, Y. (2015). Interdisciplinary competences and their status role in the system of higher professional education. Procedia - Social and Behavioral Sciences, 206, 429 - 433. https://www.doi.org/10.1016/j.sbspro.2015.10.078

Larrain, A. y González, L. (2007). Formación universitaria por competencias. Recuperado de https://www.researchgate.net/publication/275275474_Formacion_universitaria_por_competencias_2007-16 
Larrea, J. y Alonso, L. (2019). Procedimiento para la evaluación de las competencias profesionales de los estudiantes de carreras universitarias. Luz, 18(2), 94-108

Martínez, P. y González, N. (2018). Las competencias transversales en la universidad: propiedades psicométricas de un cuestionario. Educación XX1, 21(1), 231-261.

Martínez, P., González, N. y González, C. (2016). Percepción de competencias y plan de mejora para la consolidación de los títulos de grado en educación: la mirada de los estudiantes. Estudios Pedagógicos, 42(3), 277-292. https://www.doi.org/10.4067//S0718-07052016000400015

Montes de Oca, N. y Machado, E. (2014). Formación y desarrollo de competencias en la educación superior cubana. Humanidades Médicas, 14(1), 145-159.

Morelos, J. y Vergara, J. (2011). Evaluación de los egresados de los programas de ingeniera industrial de Barranquilla y su impacto sobre el medio. Revista Escenarios, 9(2), 70-76.

Neri, J. y Hernández, C. (2019). Competencias clave en el ámbito laboral- Análisis de la percepción estudiantil del área de ingeniería. Remai, 5(1), 30-39.

Palma, M., Ríos, I. \& Guerrero, D. (2012). Higher education in industrial engineering in Perú: Towards a new model Based on Skills. Procedia - Social and Behavioral Sciences, 46, 1570-1580. https://www.doi.org/10.1016/j.sbspro.2012.05.342

Palmer, A., Montaño, J. y Palou, M. (2009). Las competencias genéricas en la educación superior. Estudio comparativo entre la opinión de empleadores y académicos. Psicothema, 21(3), 433-438.

Paton, R. \& Wagner, R. (2014). Management education makes a difference: enhancing german engineering performance. Long Range Planning, 47, 277-298. https://www.doi.org/10.1016/j.lrp.2012.06.002

Rial, A. (2007). Diseño curricular por competencias: el reto de la evaluación. Recuperado de https://dugi-doc.udg.edu/bitstream/handle/10256/819/Antonio_Rial.pdf?sequence=1\&isAllowed=y

Rico-Reintsch, K. I. (2019). Uso de autoevaluación docente como herramienta innovadora para el mejoramiento de las asignaturas universitarias. Revista CEA, 5(10), 69-81.

Ronquillo, L., Cabrera, C. y Barberán, J. (2019). Competencias profesionales: desafíos en el proceso de formación profesional. Opuntia Brava, 11(Especial 1), 1-12. https://www.doi.org/10.35195/ob.v11iEspecial.653

Ruiz, A., Gallardo, J., Navarro, E. \& Cerón, M. (2019). Project-led-education experience as a partial strategy in first years ofengineering courses. Education for Chemical Engineers, 29, 1-8. https://www.doi.org/10.1016/j.ece.2019.05.004 
Salcines, I., González, N., Ramírez, A. y Martínez, L. (2018). Validación de la escala de autopercepción de competencias transversales y profesionales de estudiantes de educación superior. Profesorado, 22(3), 31-51. https://www.doi.org/10.30827/profesorado.v22i3.7989

Shamshina, I. (2014). Professional competences necessary for the bachelor-degree-holding engineer specialising in engineering industries. Pacific Science Review, 16(2), 85-88. https://www.doi.org/10.1016/j.pscr.2014.08.018

Saorín, J., Díaz, D., Bonnet, A., Carbonell, C., Meier, C. \& De la Torre, J. (2017). Makerspace teaching-learning environment to enhancecreative competence in engineering students. Thinking Skills and Creativity, 23, 188-198. https://www.doi.org/10.1016/j.tsc.2017.01.004

Silva, H. y Mazuera, A. (2019). ¿Enfoque de competencias o enfoque de capacidades en la escuela? Revista Electrónica de Investigación Educativa, 21(especial 17), 1-10. https://www.doi.org/10.24320/redie.2019.21.e17.1981

Tejada, J. y Ruiz, C. (2016). Evaluación de competencias profesionales en Educación Superior: Retos e implicaciones. Educación XX1, 19(1), 17-38. https://www.doi.org/10.5944/educXX1.12175

Tirado, L., Estrada, J., Ortiz, R., Solano, H., González, J., Alfonso, D., Restrepo, G., Delgado, J. y Ortiz, D. (2007). Competencias profesionales: una estrategia para el desempeño exitoso de los ingenieros industriales. Revista Facultad de Ingeniería Universidad de Antioquia, (40), 123-139.

Valera, R. (2010). El proceso de formación del profesional en la educación superior basado en competencias: el desafío de su calidad, en busca de una mayor integralidad de los egresados. Civilizar, 10(18), 117-134.

Vera, M., Álava, S. y Basurto, G. (2019). El desempeño profesional: bases de la formación de calidad. Opuntia Brava, 11(1), 224-234. https://www.doi.org/10.35195/ob.v11i1.716

Villarroel, V. y Bruna, D. (2014). Reflexiones en torno a las competencias genéricas en educación superior: Un desafío pendiente. Psicoperspectivas, 13(1), 23-34.

Walpole, R., Myers, R. y Myers, S. (2012). Probabilidad y estadística para ingeniería y ciencias. Naucalpan de Juárez, Estado de México: Pearson Educación de México.

Zambrano, A. (2014). Practicas evaluativas para la mejora de la calidad del aprendizaje: un estudio contextualizado en La Unión, Chile. (Tesis de Doctorado). Universidad Autónoma de Barcelona, España.

Zúñiga, A., Leitón, R. y Naranjo, J. (2014). Del sistema educativo tradicional hacia la formación por competencias: Una mirada a los procesos de enseñanza aprendizaje de las ciencias en la educación secundaria de Mendoza Argentina y San José de Costa Rica. Revista Eureka, 11(2), 145-159. 